\title{
Analysis of the early therapeutic effects and healing outcomes of segmental bone defects in the ankle joint treated with the induced membrane/wrapped bone grafting technique
}

\section{Wenbin Zhao}

Wuhan No.1 Hospital

Maopeng Wang

Three Gorges University Renhe Hospital

\section{Xingshi Lin}

Zhumadian Chinese Medicine Hospital Affiliated to Henan University of Traditional Chinese Medicine

\section{Feng Tu}

Wuhan No.1 Hospital

Dongfeng Zhao ( $\sim$ doczhaodf01@163.com)

Jiayuguan First People's Hospital https://orcid.org/0000-0001-7514-9254

\section{Research}

Keywords: Induced membrane technique, Ankle joint, Bone defect, Wrapped bone grafting

Posted Date: April 2nd, 2020

DOI: https://doi.org/10.21203/rs.3.rs-19889/v1

License: (c) (i) This work is licensed under a Creative Commons Attribution 4.0 International License.

Read Full License 


\section{Abstract}

\section{Background}

The current research explores the early therapeutic efficacy and healing outcomes of segmental bone defects in the ankle joint treated with induced membrane technique.

\section{Methods}

A segmental bone defect model of ankle joint was first constructed by removing $2 \mathrm{~mm}$ bone from the ankle joint of the rat, and then the induced membrane treatment was performed in two steps: the first step was to implant polymethyl methacrylate bone cement after thorough debridement, followed by the second step to remove bone cement after membrane formation and to replace with the rat's autologous cancellous bone. The physiological indicators (body temperature and body weight) of the rats and the TNF- $a$ and CRP in the blood were monitored post-surgery, and the efficacy was analyzed based on the above combining Micro-CT and X-ray analysis. Postoperative histological analysis of the tissue morphology of partial induced membrane was performed in rats at $2,4,6$, and 8 weeks to evaluate the tissue status at the sites of bone defect.

\section{Results}

Results showed that the rats survived well after operation: the body temperature slowly decreased, and the CRP was also gradually reduced to normal; the 12-week Micro-CT and palpation indicated a satisfying bone healing trend; histological studies found calcified tissue in the second week post-operation, and vascular network was established in the induced sites at 8 weeks.

\section{Conclusion}

The study proves that the induced membrane technique can effectively treat segmental bone defects of ankle joint, and is less prone to infection.

\section{Background}

Segmental bone defects (shorted as SBD) are mainly caused by trauma, infection, and tumor, and are one of the common clinical problems encountered in surgeries [1-3]. The current treatment methods generally include Llizarov technology, vascularized bone graft, together with quite a number of allografts and tissue engineering techniques [4, 5]. In 2000, Masquelet et al. [6] used polymethyl methacrylate (PMMA) bone cement and autologous cancellous bone grafting to repair bone defects in the limbs, which was later referred to as the induced membrane technique (the IM technique; also, the Masquelet technique).

First proposed by Masquelet, the IM technique used bone cement to induce membrane formation and then autologous cancellous bone graft to fill the defect, achieving satisfactory results. Since its 
introduction, this technology has been widely used in clinical practice. It is based on theories that various tissue cells grow in the defects at different speeds, thus the formed membrane will act as a biological barrier to the surrounding soft tissues, providing a relatively stable environment for bone tissue to repair and regenerate. There are two steps in the IM procedure: first, debridement of the lesion site and implantation of PMMA bone cement to induce membrane; second, removal of the bone cement and embedment of the cancellous bone in the membrane at $6-8$ weeks. The success rate of this technique for repairing bone defects is $88-100 \%$, and the repaired bone defect can reach $25 \mathrm{~cm}[7,8]$. At present, the IM technique is becoming one of the effective methods to treat bone defects, especially infectious bone defects, and is gradually widely used in plastic surgery [2, 9-11]. It has been reported that the IM technique can be used to treat traumatic bone defects, bone defects after malignant tumor resection, and chromosomal nic osteomyelitic bone defection (OBD). The IM technique has shown apparent advantages including short treatment cycle, limited complications, simple operation and satisfactory clinical outcomes [12].

The current study established a model of SBD of ankle joint in rats and conducted early efficacy analysis to provide a basis for applying the IM technique in the SBD of ankle joint.

\section{Materials And Methods}

\subsection{Laboratory Animals}

Twelve-month-old Sprague-Dawley male rats, with an average weight of 800 grams (purchased from Shanghai Lab Animal Research Center); rats were given 12 hours of light (6: 00-18: 00) daily, and were provided with sufficient food and water. Authors state that they complied with the tenets of the Declaration of Helsinki or the NIH statement for the use of Animals in this study, and this research was approved according to the relevant laws and institutional regulations.

\subsection{Establishment of experimental model and grouping}

Ankle joint SBDs were created in the right hind limb of the rat. The rats were fasted for solids for 12 hours and for liquids for 6 hours before surgery. A combination of ketamine $(100 \mathrm{mg} / \mathrm{kg})$ and xylazine $(10 \mathrm{mg}$ $/ \mathrm{kg}$ ) were injected intraperitoneally to anesthetize the rates. The rats were lay supine and their limbs were fixed. Strict disinfection was done using povidone-iodine and sterile surgical towels were placed. A $20 \mathrm{~mm}$ longitudinal incision was made in the middle of the rat's ankle, and fascia and muscles were exposed in sequence. The middle portion of the joint was separated bluntly and a wire saw was used to cut off 2-mm bone (including periosteum) from the middle ankle bone (the self-healing limit of the rat is $2 \mathrm{~mm}$ ). After washing with $0.9 \%$ sodium chloride solution, the medullary cavity at the fracture end was closed with bone wax to reduce blood in the medullary cavity. Then the soft tissues of each layer of the fascia and muscles were sutured in sequence, and finally a sterile dressing was applied. The rats were fasted for solids and liquids for 4 hours post-operation. Each rat was fed independently. Penicillin was injected intramuscularly for 3 consecutive days at 200,000 units per day. 
The rats were divided into 3 groups, namely the control group and the model \& operation group. There were 3 rats in the control group (healthy rats) and 9 in the model group (SBD model rats) (3 for detecting physiological and biochemical indicators; 3 for histological identification; 3 for X-ray examination and palpation); the operation group (SBD rats treated with IM) had a total of 18 rats (3 for physiological and biochemical indicator detection and Mirco-CT examination; 12 for histological identification; 3 For X-ray examination and palpation).

\subsection{The IM surgery procedure}

The IM surgery was performed in two steps. After the model operation, the first step was done postradical debridement by implanting antibiotics and bone cement. Debridement was implemented to expose the normal cortical bone interface, and bone defects were fixed with intramedullary nails. Mixed vancomycin and PMMA bone cement at a ratio of 1:20 were placed into the bone defect to connect the two ends and the incision was closed. If infection occurred within 8 weeks, repeat thorough debridement. In the second step, bone defects were reconstructed by cancellous bone transplants (taken from rat tail). The induced membrane of the bone defect was cut longitudinally and the bone cement was carefully removed. A loose cancellous bone graft was implanted at the defect. The incision was closed, washed with saline and disinfected with iodophor.

\subsection{Bone sample collection and efficacy evaluation}

Animals were sacrificed by intraperitoneal injection of pentobarbital. Twelve weeks after the second operation, the ankle bones of the rats were collected for X-ray and palpation examination. Any defect or gap found by palpation was considered nonunion. Ankle bones without significant gap were evaluated for healing efficacy by axial percussion or pull with sufficient force; the absence of loosening was considered as successful osseointegration and reconstruction.

Scanning was performed on a Micro-CT (SkyScan 1172, Belgium) instrument. The scanning parameters were as follows: $180^{\circ}$ rotation, $0.4^{\circ}$ step, scanning resolution of $12 \mu \mathrm{m}$, tube voltage of $60 \mathrm{kV}$, tube current of $120 \mu \mathrm{A}$, exposure time of $700 \mathrm{~ms}$, and 0.5 aluminum filter. After the scanning, a cross-sectional view of the specimen was obtained using the reconstruction software NRe-con (V1.14.4) that came with the device. CTan (V1.14.4) software was used to analyze the data. 200 layers (401 layers in total) both above and below the fracture line with the fracture line as the center were selected as the region of interest (ROI) to complete the image binarization (threshold 80-255). Then analysis was performed and the following indicators generated from the ROI were compared among various groups: the trabecular bone volume (BV), total ROI volume (TV), bone volume/ROI volume fraction (BV/TV), trabecular bone pattern factor (Tb.Pf), trabecular bone separation (Tb.Sp), and bone mineral density (BMD).

\subsection{Histological examination}

Three rats in the operation group were sacrificed at 2, 4, 6, and 8 weeks for histological examination. A $7 \mu \mathrm{m}$ lateral cross-section sample was obtained using Shandoncryotome ${ }^{\circledR}$ FE (Shandon cryotome ${ }^{\circledR} \mathrm{FE}$; Thermo Electron Corporation, Waltham, MA, USA) at $7^{\circ} \mathrm{C}$ and it was pasted on SuperFrost ${ }^{\circledR}$ Slides 
(Menzel-Gläser, Brunswick, Germany). After drying at room temperature, the sections were sealed and stored at $-80^{\circ} \mathrm{C}$. The sections were stained with hematoxylin and eosin (H\&E) to analyze general tissue morphology, and then stained with Alizarin Red S ( $40 \mathrm{mmol} / \mathrm{j}, \mathrm{pH} 4.2)$ and Von Kossa ( $5 \%$ silver nitrate) for 30 minutes in the dark to identify mineralization in the tissue. The alkaline phosphatase (ALP) staining procedure was performed using an ALP staining kit (Takara Bio Inc., Shiga, Japan) according to the kit instructions.

\subsection{Statistical Analysis}

The data were analyzed using statistical software SPSS17.0. Measurement data were expressed as mean \pm standard deviation ( $x \pm s$ ), and comparison between groups was performed by $t$ test. The difference was statistically significant at $P<0.05$. All BMD data were processed by statistical software $S A S$. The BMD values in the observation period after fracture reduction of each end was used as a selfcontrol, and paired t-test was performed for each portion using a pre-and post- self-controlled method.

\section{Results}

\subsection{Rat survival after the IM treatment}

The decrease in the body temperature (BT) of rats in the operation group from the first week to the eighth week post-surgery was related to the number of days post-treatment $(r=0.981, P<0.001)$. With the increase of time, the BT of rats gradually returned to normal. There was no significant correlation between BT and post-treatment time in the control and model groups $(r=0.092, P>0.05) .7$ weeks after treatment, the $\mathrm{BT}$ of the rats in the operation group was significantly lower than that in the model group $(P<0.05)$ (Fig. 2).

\subsection{Changes of TNF- $a$ and CRP levels in rats at different time points}

C-reactive protein (CRP) can be used for the determination of the level of inflammation and the preliminary determination of viral or bacterial infection. Tumor necrosis factor (TNF-a) is negatively correlated with osteoblast proliferation and differentiation. During the whole treatment process, the levels of CRP and TNF- $a$ in the model group were significantly higher than those in the control group $(P<0.05)$. Compared with the model group, the levels of CRP and TNF- $a$ in the rats treated with the IM technique were significantly reduced $(P<0.05)$ (Fig. 3).

\subsection{Comparison of Micro-CT parameters pre- and post- operation in the rat ankle joints}

The BV, TV, BV/TV and Tb.Pf of selected ROls were calculated using software (Fig. 4A, 4B, and 4C). Compared with preoperative values, postoperative BV, BV/TV (\%) and Tb.Pf (mm-1) significantly 
increased In the 12 th week post-operation $(P<0.05$ or 0.01$)$, while Tb.Sp $(\mathrm{mm})$ was significantly reduced $(P<0.05)$.

Analysis of the rats during the healing period (Fig. 4D) revealed that the overall (R3) BMD of the fracture end gradually increased from a lower value and reached its maximum at 8-10 weeks, showing a positive development trend and statistical significance $(P<0.001)$. During this period, the BMD value in the fracture area (R3) gradually increased generally; there were "jumps" in the BMD percentage trend 1 week to 4 weeks postoperatively, and it increased by an average of $1 \%$ every week from the 6 to 10 weeks. All these clearly indicated a satisfying trend of fracture healing during this time.

\subsection{Study of the IM}

After hematoxylin and eosin ( $\mathrm{H} \& \mathrm{E})$ staining (magnification $\times 10$ ), the IM was observed under a microscope, and its tissue structure was observed (Fig. 5). Three different regions were identified in the IM during 2-8 weeks. The first was a thin layer of cells (5 to 7 cells thick) with abundant cell layers which were in contact with PMMA.Within 2-8 weeks of PMMA insertion, this region was observed in all IMs (double yellow arrow). The second region gradually appeared 4 weeks after the insertion of PMMA (cyan double arrows), consisting of a fiber network oriented parallel to PMMA. Compared to the previous region, this part of the inducted membrane had a lower cell density, and its cell size increased over time, indicating that the cells in the membrane matured progressively in 2-8 weeks. Finally, the region in contact with the muscles (pink double arrow) was thicker than the other two regions. This region at the interface between the membrane and the surrounding muscles was composed of loose, messy tissue, containing a large network of blood vessels.

Alizarin Red $S$ and Von Kossa staining (magnification $\times 20$ ) found calcium phosphate and calcium carbonate spots in the areas where the IM was in contact with PMMA (yellow double arrow and cyan double arrow), indicating that the IMs at 2-8 weeks all showed mineralization. Calcium carbonate deposits were stained red (red arrows) by alizarin red $S$ and black (blue arrows) by Von Kossa. Alkaline phosphatase (ALP) staining revealed a dynamic maturation process of the IM (Yellow Brother and Cyan Double Arrow). Although the IM was all positive for ALP staining (dark purple) at each stage, the IMs 2- to 8-week-old showed different staining patterns. The parts on the left were in contact with PMMA: the 4week-old IMs were more obviously stained by ALP than in the other groups. At 6 and 8 weeks, the ALP staining on the IM was relatively lighter and overlapped with the previous hematoxylin and eosin stains (cyan double arrow).

\subsection{The IM technique repairing SBD in rats}

X-ray examination was performed on the ankle joints of the rats in the model group and the operation group at 12 weeks post-operation. The model group rats without IM treatment showed bone fracture and no healing. The ankle joints of the rats in the operation group showed signs of bone formation. During palpation, the ankle bones of the rats in the operation group showed strong osseointegration, while those of the model group had obvious gaps. 


\section{Discussion}

Traditional treatments, such as open bone grafting, vacuum-sealed drainage and antibiotic irrigation and perfusion, have a series of disadvantages, including complicated operations, poor treatment outcomes, which will lead to more complications, prolonged treatment period and poor patient acceptance. Masquelet first proposed the IM technique, in which debridement is applied at the site of bone defects followed by implantation of antibiotic bone cement, and bone reconstruction is performed using autologous cancellous bone graft after IM formation. After the antibiotic and bone cement are mixed and applied, the local drug concentration is increased, thereby achieving a sustained release effect and improving the treatment effect. Bone cement protects damaged bone tissue, thereby reducing the risk of fractures after radical debridement. The operation process is simple and the treatment effect is good. There are various researches on the clinical treatment of SBD using the IM technology and satisfactory results have been reported $(13,14)$. Pelissier et al. $(15)$ investigated the expression of various cytokines during bone formation using rabbit inducible membrane technology in rabbits. Aho et al. (16) performed IM surgery on patients and analyzed the components of the IM under different conditions.

In this study, a rat model of ankle bone defect was established and treated with the IM technology. Their results showed that all rats reached complete bone healing after the IM therapy within a healing period of only 12 weeks, and all were able to move weight-bearing. The body weight (BW), BT, and the number of bacteria in the wound of the rats decreased over time after the IM treatment. Weight loss may be caused by loss of appetite due to pain during model establishment and treatment. The decrease in the BT of rats and the reduction of bacteria in the wound indicated that after implantation of antibiotic bone cement, local high concentrations of antibiotics can be obtained around the bone defect, thereby effectively inhibiting the growth of various bacteria and reducing the incidence of postoperative infection.

A dense collagen network (thick layer of high-density cells) was observed in the second week after the first stage of surgery (membrane induction), confirming the biological tissues found in sheep (15) and rabbits (16). In humans, the IM has been found calcified even before transplantation (17), which may indicate that the IM can promote the integration and mineralization of the transplanted bone. In the model used in this study, the IM showed calcification in the second week after PMMA was inserted, and the calcified area was discontinuous in the membrane. Cells grew clinging to PMMA within 2-8 weeks, and rich blood vessels were gradually built in the eighth week. After ALP staining, osteoblasts were observed in the IM, confirming the evidence previously reported by Viateau using anti-Cbfa1 markers in a sheep model. Relevant researches show that the bone self-healing limit of rats is $2 \mathrm{~mm}$. At the 4 th week after the second surgery (intramembrane grafting), the rats without the IM treatment still showed bone nonunion. Contrarily, the rats treated were able to act weight-bearing. Bone samples were collected for Xray and palpation examination, which revealed that the rats that underwent two operations exhibited satisfactory bone formation.

\section{Conclusion}


In conclusion, the results from our study by establishing a rat SBD model and then treating the rats with the IM technique showed that the bone healing effect was significant post-surgery. In addition, the treated rats were free of wound infections and complications. Our study provides the basis and reference for applying the IM technique in the treatment of ankle bone defects.

\section{Declarations}

\section{Ethics approval and consent to participate}

Authors state that they complied with the tenets of the Declaration of Helsinki or the NIH statement for the use of Animals in this study, and this research was approved according to the relevant laws and institutional regulations.

\section{Consent for publication}

Not applicable

\section{Availability of data and materials}

All data generated and analyzed during this study are included in this published article.

\section{Competing interests}

The authors declare that they have no competing interests.

\section{Funding}

Not applicable.

\section{Authors' Contributions}

Project administration: DFZ; Writing - review \& editing: WBZ, MPW; Data curation: FT; Methodology: XSL; Resources: DFZ. All authors have read and approved the manuscript.

\section{Acknowledgement}

Not applicable.

\section{References}

[1] Mariappan $\mathbb{I}$ Maddileti S $\triangle$ Joseph $P \bigotimes$ et al. Enriched Cultures of Retinal Cells From BJNhem20 Human Embryonic Stem Cell Line of Indian Origin[J]. Investigative Ophthalmology \& Visual Science $2015 \rrbracket$ 56(11):6714-23. 


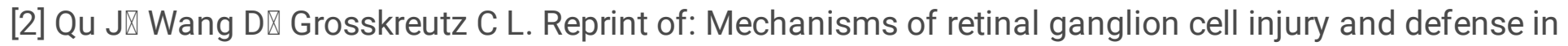

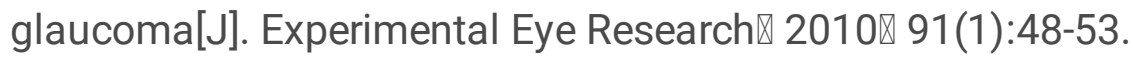

[3] Casson $R J \otimes$ Chidlow $G \otimes$ Wood $J P \unrhd$ et al. The effect of retinal ganglion cell injury on light-induced photoreceptor degeneration.[J]. Investigative Ophthalmology \& Visual Science区 2004囚 45(2):685-693.

[4] Mohan K囚 Kecova $\mathrm{H} \otimes$ Hernandezmerino $\mathrm{E} \otimes$ et al. Retinal Ganglion Cell Damage in an Experimental Rodent Model of Blast-Mediated Traumatic Brain Injury[J]. Investigative Ophthalmology \& Visual Science区 2013凶54(5):3440-3450.

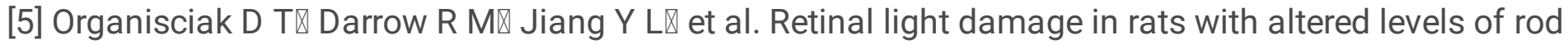
outer segment docosahexaenoate.[J]. Invest Ophthalmol Vis Sci囚 1996ه 37(11):2243-2257.

[6] Song W K囚 Park K M囚 Kim H J『 et al. Treatment of Macular Degeneration Using Embryonic Stem Cell-

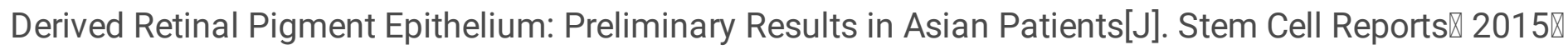
4(5):860-872.

[7] Suzuki $\mathrm{T} \otimes$ Mandai $\mathrm{M} \otimes$ Akimoto $\mathrm{M} \otimes$ et al. The simultaneous treatment of MMP-2 stimulants in retinal

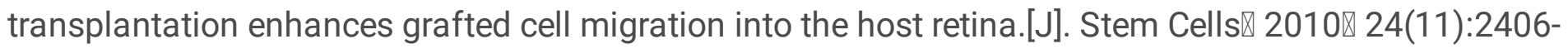
2411.

[8] Lund R D Kwan A S $₫$ Keegan D J『 et al. Cell transplantation as a treatment for retinal disease.[J]. Progress in Retinal \& Eye Research囚 2001ه 20(4):415-449.

[9] Klassen H. Stem cells in clinical trials for treatment of retinal degeneration[J]. Expert Opin Biol Ther叉 2016ه 16(1):7-14.

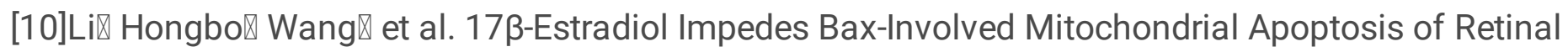
Nerve Cells Induced by Oxidative Damage via the Phosphatidylinositol 3-Kinase/Akt Signal Pathway[J]. Journal of Molecular Neuroscience $\mathbb{2}$ 2013囚 50(3):482-493.

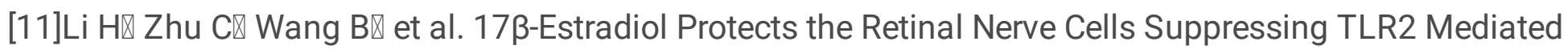
Immune-Inflammation and Apoptosis from Oxidative Stress Insult Independent of PI3K[J]. Journal of Molecular Neuroscience $2016 \rrbracket$ 60(2):195-204.

[12]Lambuk $L \bigotimes$ Jafri A J A $A$ lezhitsa $\mathbb{\|}$ et al. Dose-dependent effects of NMDA on retinal and optic nerve

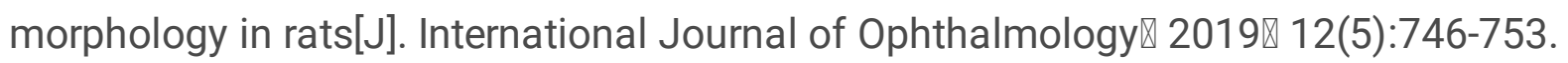

[13]Gao Y $\otimes H u Y ~ Y L i R ~ S \otimes$ et al. Cattle encephalon glycoside and ignotin injection improves cognitive impairment in APPswe/PS1dE9 mice used as multitarget anti-Alzheimer's drug candidates[J]. 20152(11): 537-548

[14]Allergic reaction to cattle encephalon glycoside and ignotin injection : 3 case reports[J]. Adverse Drug Reactions Journal $\mathbf{2 0 0 4 \rrbracket}$ 10(3):245-257. 


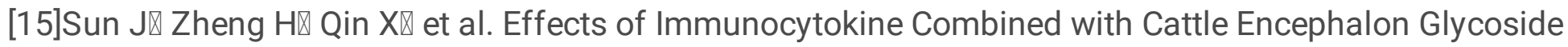
and Ignotin on CTGF区 HO-1 and NT-3 in Patients with Type 2 Diabetic Peripheral Neuropathy[J]. Iranian Journal of Public Health $₫ 2017 \rrbracket$ 46(12):1632-1638.

[16]Aizu $Y \otimes$ Katayama $H \otimes T a k a h a m a ~ S \otimes$ et al. Topical instillation of ciliary neurotrophic factor inhibits retinal degeneration in streptozotocin-induced diabetic rats[J]. Neuroreport $2003 \rrbracket$ 14(16):2067-2071.

[17]Intraperitoneal administration of adipose tissue-derived stem cells for the rescue of retinal degeneration in a mouse model via indigenous CNTF up-regulation by IL-6[J]. Journal of Tissue

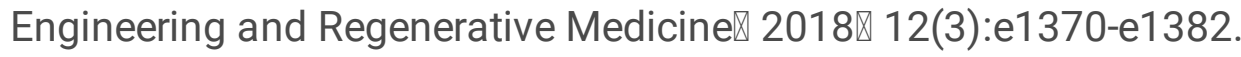

[18]Garcia-Delgado A B $\otimes$ Lourdes Valdés-Sánchez $\bigotimes$ Calado S M $\bigotimes$ et al. Rasagiline delays retinal degeneration in a mouse model of retinitis pigmentosa via modulation of $\mathrm{Bax} / \mathrm{Bcl}-2$ expression[J]. Cns

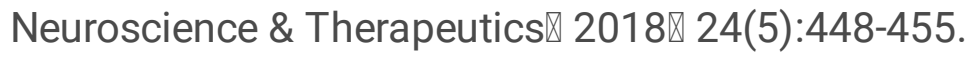

\section{Figures}

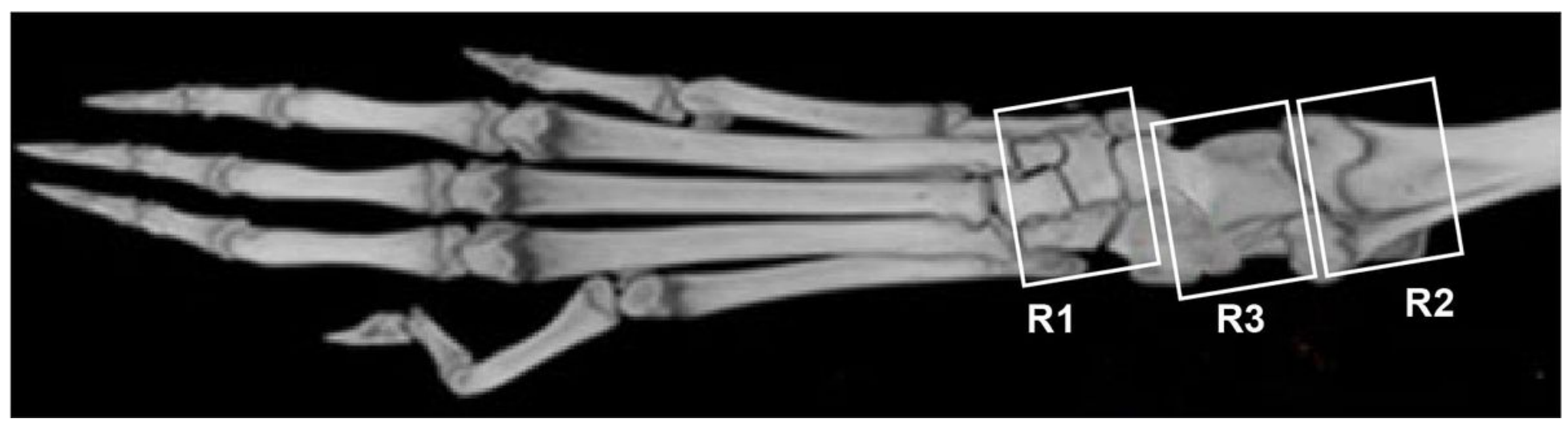

Figure 2

Schematic diagram of methods for selecting $\mathrm{ROI}$ regions

A

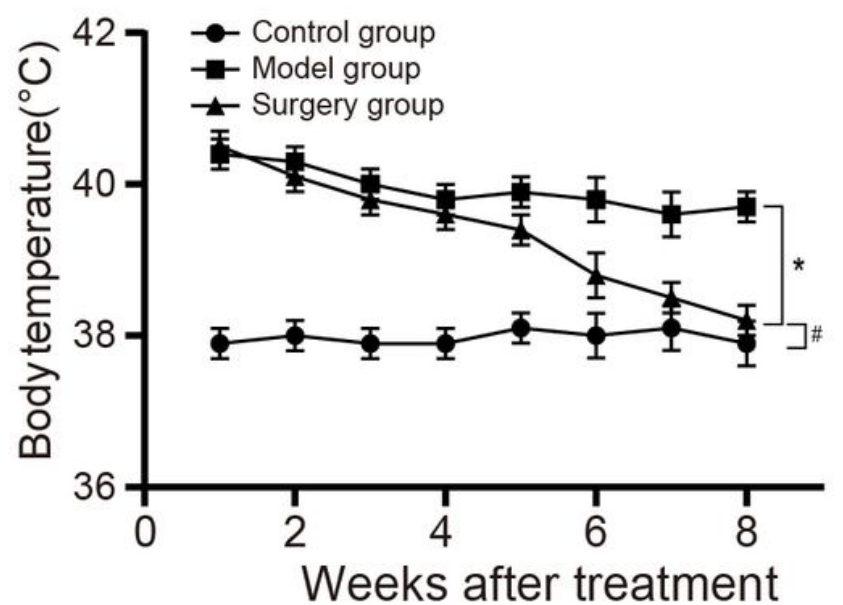

B

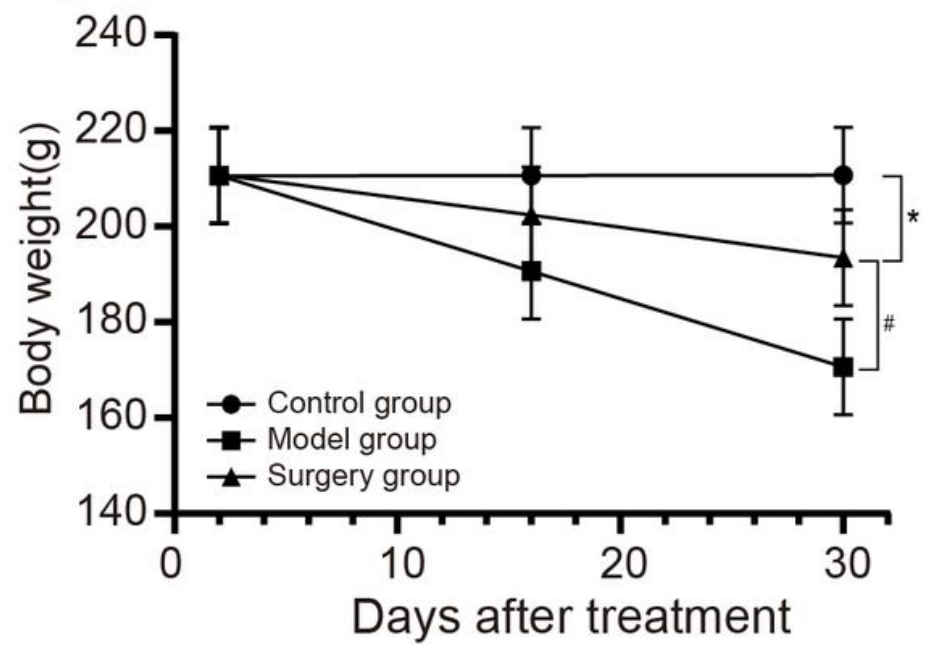


Figure 4

Changes in physiological indicators of the three groups. (A) Changes in body temperature of the three groups of rats. (B) Changes in body weight of the three groups of rats. ( $P<0.05$, compared with the control group. $\# \mathrm{P}<0.05$, compared with the model group)

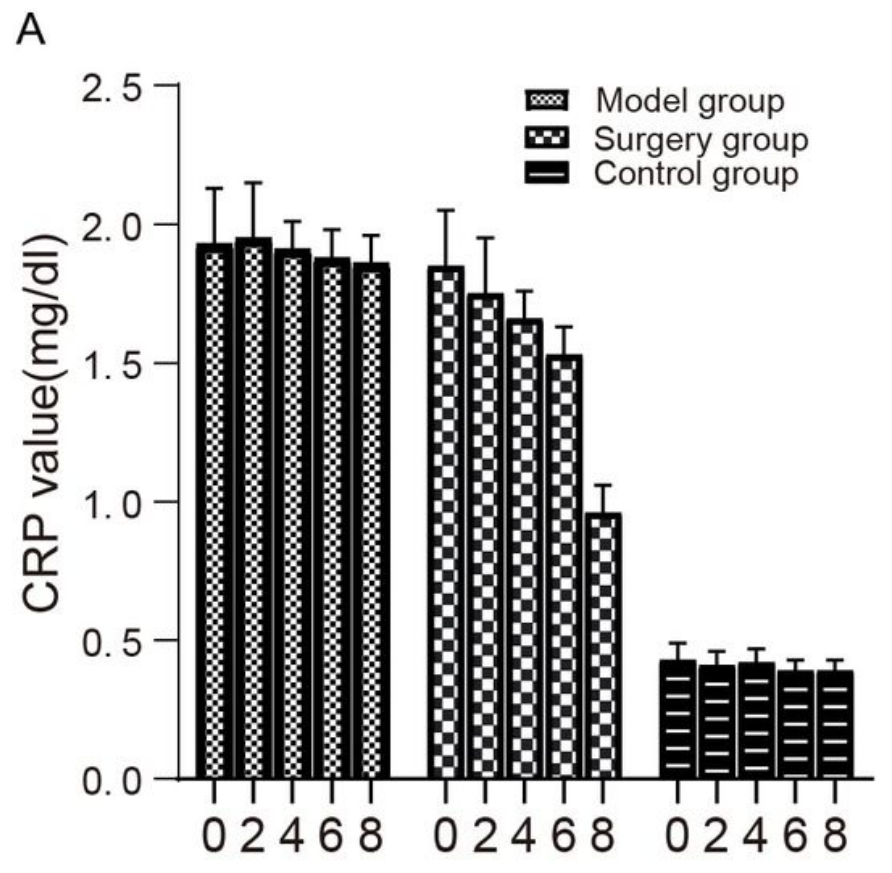

Weeks after treatment

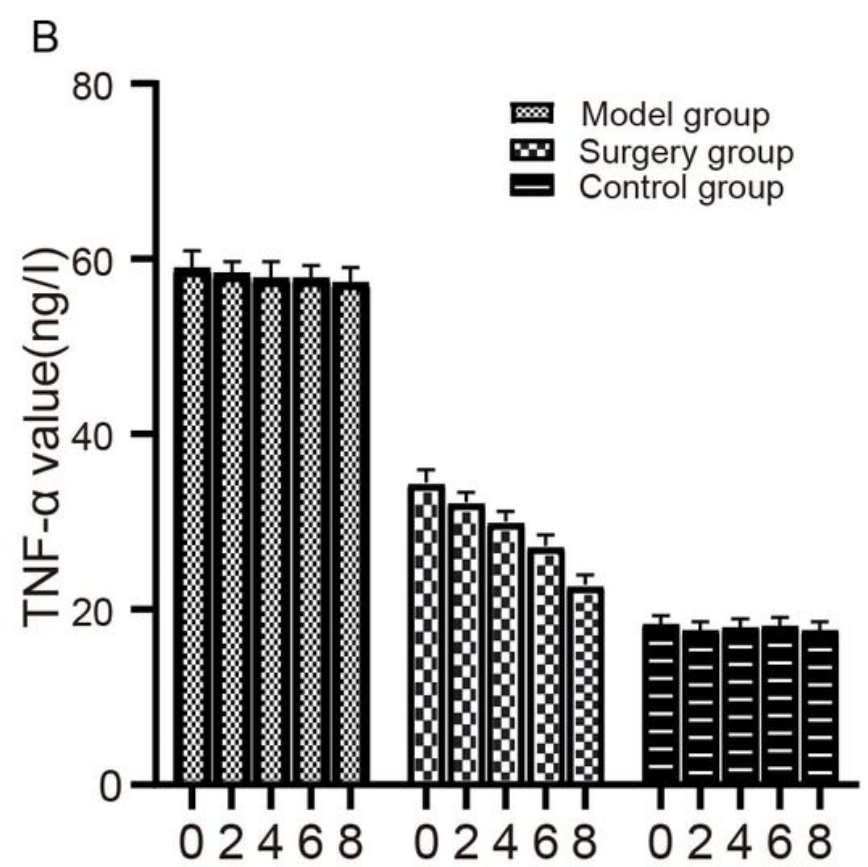

Weeks after treatment

Figure 6

Changes in blood indicators of the three groups of rats. (A) Comparison of serum CRP levels at different time points in rats (B) Comparison of serum TNF-a levels at different time points in rats 

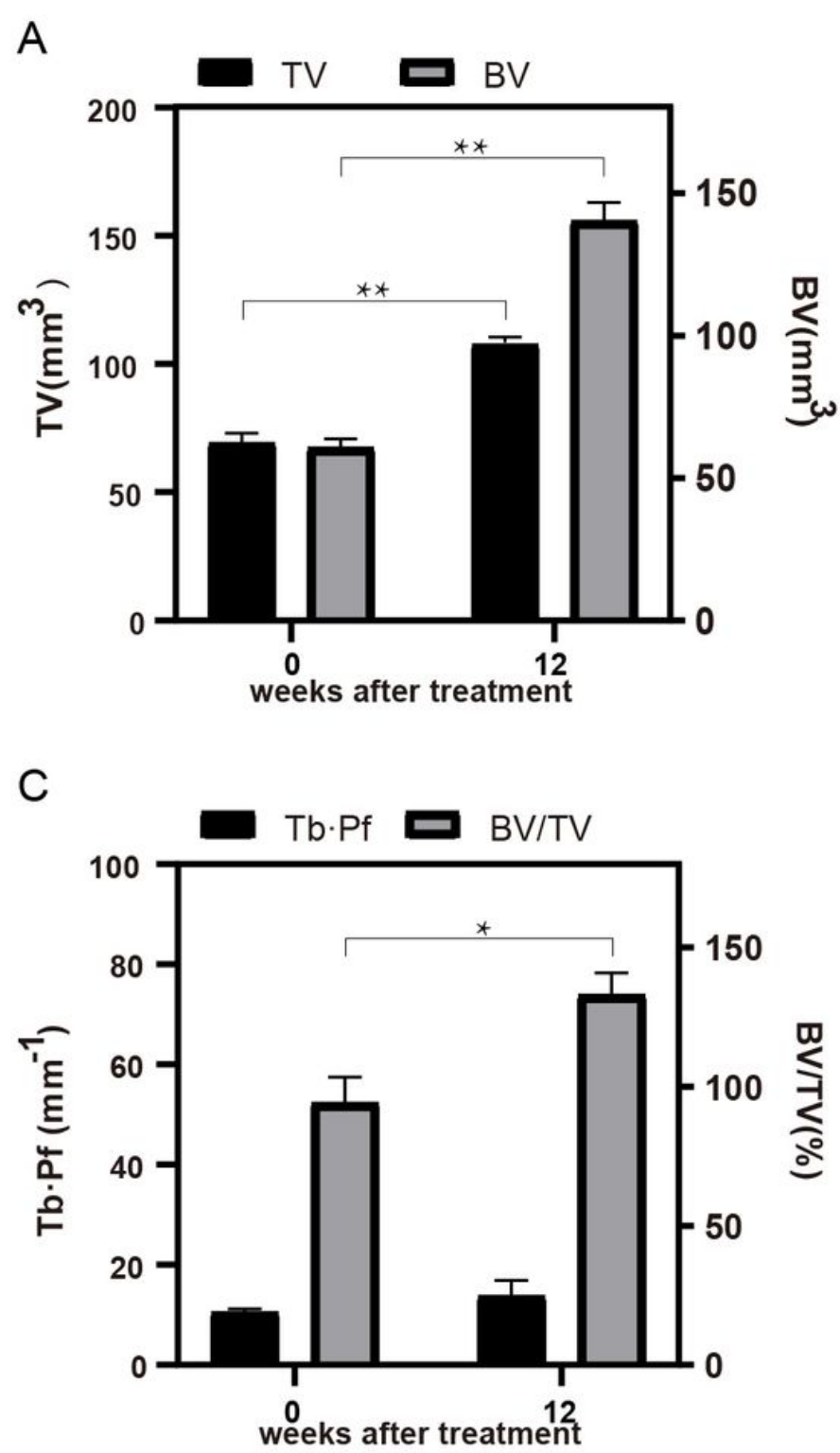

B
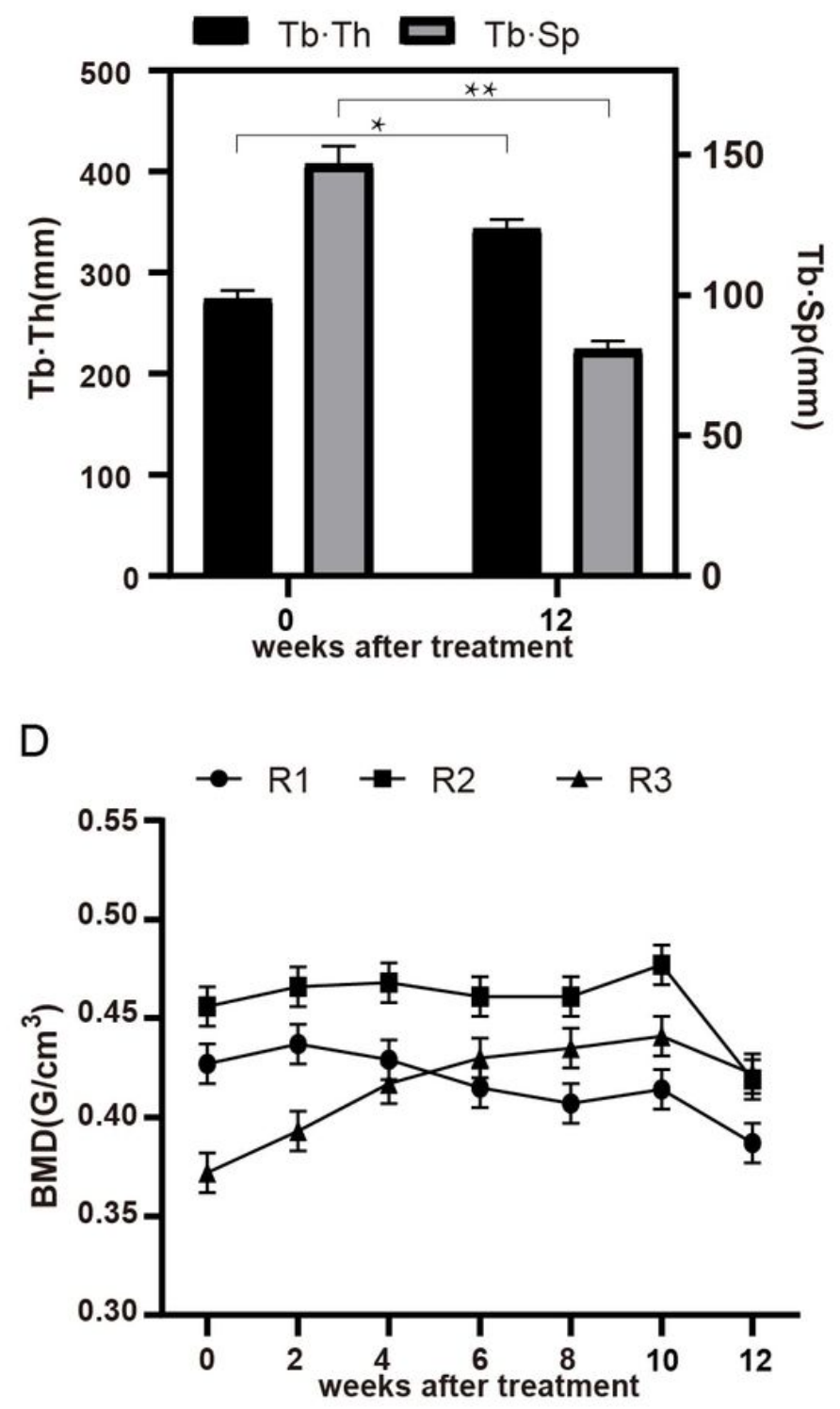

Figure 8

Bone healing status (A-C) Comparison of Mirco-CT parameters of the ankle D: Comparison of BMD value in analysis areas. (Degree of anisotropy 0 weeks: $1.88 \pm 0.19 ; 14$ weeks: $1.6 \pm 0.1 . p<0.05 \varpi^{\star \star} p<0.01$ ) 


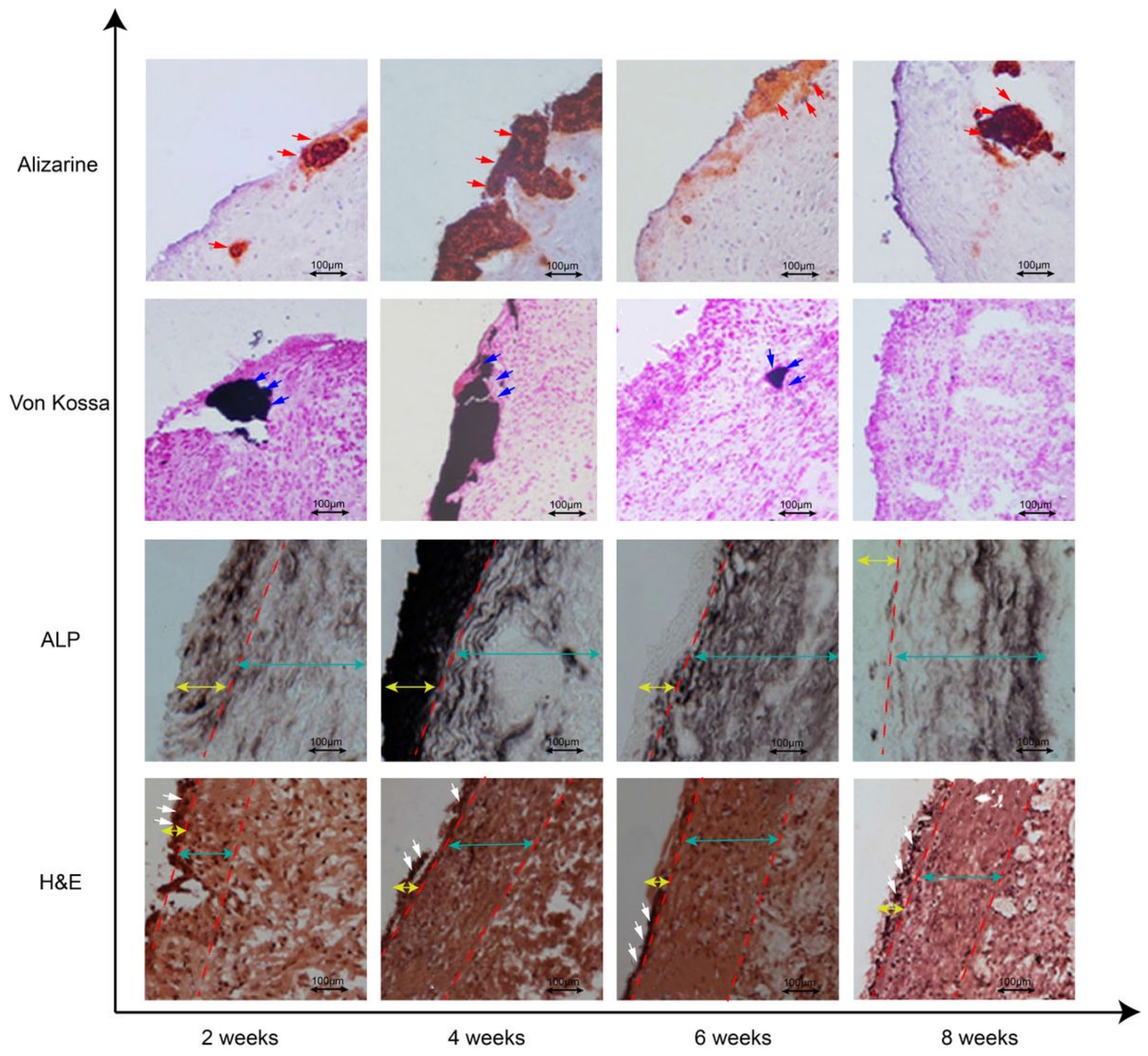

Figure 10

Induced membrane (IM) staining results. The range between the yellow double arrows is the thin layer of high-density cells in the outermost portion of IM at 2-8 weeks; the range between the blue double arrows is the low-density cell layer, and the range between the pink double arrows is a layer of organized cells and vascular network. PMMA lay left to the IM before it was removed (the results in the pictures all represent more than two independent experiments.) 